\title{
MINIATURE HIGH-LET RADIATION SPECTROMETER FOR SPACE AND AVIONICS APPLICATIONS
}

\author{
E.G. Stassioopoulos ${ }^{1}$, Craig A. Stauffer ${ }^{2}$, G.J. Brucker ${ }^{3}$
}

\begin{abstract}
This paper reports on the design and characterization of a small, low power, and low weight instrument, a High-LET Radiation Spectrometer (HiLRS), that measures energy deposited by heavy ions in microelectronic devices. The HiLRS operates on pulse-height analysis principles and is designed for space and avionics applications. The detector component in the instrument is based on large scale arrays of $p-n$ junctions. In this system, the pulse amplitude from a particle hit is directly proportional to the particle LET. A prototype flight unit has been fabricated and calibrated using several heavy ions with varying LETs and protons with several energies. The unit has been delivered to the Ballistic Missile Defense Organization (BMDO) c/o the Air Force Research Laboratory in Albuquerque, NM, for integration into the military Space Technology Research Vehicle (STRV), a US-UK cooperative mission. Another version of HiLRS is being prepared for delivery in April to the Hubble Space Telescope (HST) project, to fly on the HST Orbital Systems Test (HOST) Platform on a shuttle mission.
\end{abstract}

\section{INTRODUCTION}

Evaluation of Single Event Effects performance of integrated circuits in the space or avionics environment has in the past been a complex, multistep process, starting with the in-flight

\footnotetext{
${ }^{1}$ NASA Goddard Space Flight Center, Greenbelt, MD 20771

${ }^{2}$ Systems Engineering and Security, Greenbelt, MD 20770

${ }^{3}$ Radiation Effects Consultants, Inc., West Long Branch, NJ 07764
} 
measurement of a spectrum of ionizing particle energy, charge, and mass. From these parameters, particle identification and a correlated Linear Energy Transfer (LET) spectrum can be derived. This measured LET spectrum is used to calculate the Single Event Effects (SEE) performance of specific circuits with known SEE cross-sections. A new approach has been developed which uses integrated circuit technology to better represent the nature of the devices under investigation, and provide a direct measurement of LET[1,2].

This alternate method employs solid state microdosimeters that measure the pulse-height spectra generated when energetic charged particles traverse $p-n$ junctions having dimensions comparable to the sensitive volume of modern microelectronics. The design is such that pulse amplitude is directly proportional to the particle LET. A compact small scale prototype of such an instrument has been developed by the Radiation Physics Office (RPO) of Goddard's Applied Engineering Technology Directorate.

The HiLRS detector is based on arrays of $p$ - $n$ junctions implemented in silicon. It uses advanced lithographic techniques developed by the microelectronic industry. The detector records the pulse-height spectra from events at individual junctions having dimensions typical of SEU-sensitive structures of modern microelectronic devices. Each junction has associated with it a sensitive microvolume such that a charge generated within that microvolume will contribute to the signal at the junction while charge generated outside will not. Proper design of the individual junctions can make the amount of energy deposited relatively insensitive to 
the direction of the incident particle. Prototypes using up to 2 million junctions have been demonstrated.

The purpose of flying such an instrument on board a satellite, rocket, or aircraft is to characterize the radiation environment in space and at avionics altitudes in terms of risk and hazard to microelectronic systems or instruments, to optimize decisions regarding when to turn instruments on or off, to provide actual flight data for improving algorithms for SEE predictions based on ground test measurements, or to assist in failure analysis during flight.

Some other benefits deriving from on-orbit or in-flight avionics measurements are: verification of models, correlation of events on a spacecraft or aircraft to predictions, aiding in the analysis of flight data, identifying possible causes of anomalies, failures or abnormal performance, enabling systems validation, characterizing solar proton events by deposition spectra behind different layers of shielding, assisting in post-flight performance evaluation of electronic components.

The HiLRS radiation monitor-microdosimeter has been designed to conform with current trends in technology development. Thus, it's most appealing attributes are low cost, light weight, low power, small size, and minimal telemetry.

\section{DESCRIPTION OF HILRS AND ITS OPERATION}

Figure 1 contains a block diagram of the components that make up the HiLRS system. A very important property of this design is that all of the support semiconductors are rad-hard parts. 
The basic particle detector is a 256K SRAM. Incident particles deposit energy in or near any of the $256 \mathrm{~K}$ p-n junctions. Bias is not applied to the junctions. The intrinsic electric field of the junction provides the mechanism to collect the particle-induced charge. The charge pulse is first handled by the charge sensitive amplifier to transfer the signal to a voltage pulse (no gain). The pulse is passed to the main amplifier where significant gain takes place. The signal is digitized by the ADC (Analog-to-Digital Converter). The LLD (Low Level Discriminator) decides whether the pulse amplitude satisfies the threshold limit and if yes, the LLD triggers the microprocessor (CPU) to manage the data flow and direct the flow in accordance with the software program.

The instrument is powered-on all the time and ready to measure the energy deposited in the arrays of microjunctions by Galactic and Solar Cosmic Rays (GCRs, SCRs) and their daughter products (neutrons, heavy ions, protons) from interactions with spacecraft or aircraft materials and atmospheric constituents (oxygen, nitrogen). If exposed to trapped Van Allen Belt protons and/or to Solar Proton Events (SPEs) during periods of solar activity, it will also detect their spallation, fractionation, and recoil products in satellite and airplanes. If power is lost, the stored data is also lost but the program is automatically reinitialized and reactivated when power returns. A backup battery to retain the data in the SRAM can be incorporated in the design to prevent loss of data. The weight of the present HiLRS is about 214 grams and the power requirements are $+5 \mathrm{~V}, 0.25$ watts and $-5 \mathrm{~V}, 0.02$ watts. Its size is $4.5^{n} \times 4.5^{n} \times 0.5^{\prime}$. The development of future units is in progress with an objective aimed at further reduction in size, weight and power. It is anticipated that this can be accomplished by combining some of 
the integrated circuits and functions. A factor of 2 to 3 reduction in size appears to be possible, depending on spacecraft requirements and the complexity of the HiLRS system. For example, modifying the system to enable direct measurements of protons by including some type of a proton detector. Figure 1 shows a simple block diagram of the HiLRS instrument.

\section{CHARACTERIZATION AND CALIBRATION}

The HiLRS detector is a $32 \mathrm{~K} \times 8 \mathrm{SRAM}$, with a die area of $0.25 \mathrm{~cm}^{2}$, a node (junction) cross section of $49 \mu \mathrm{m}^{2}(x=y=7 \mu \mathrm{m})$, a sensitive volume depth of $14 \mu \mathrm{m}$ (estimated), and an EPI layer of $5 \mu \mathrm{m}$ (assumed).

The instrument was calibrated with heavy ions at the Tandem Van de Graaff facility of the Brookhaven National Laboratory in Upton, Long Island, NY. Five ion species were used, as listed in Table 1. The corrected energies, LETs, and penetration ranges of these particles, incident on the sensitive volume after passing through the $5 \mu \mathrm{m}$ EPI layer, are also given.

All exposures were performed at normal incidence with the detector unbiased. Figure 2 contains a plot of the basic calibration curve for the HiLRS that indicates the value of LET versus channel number. The five heavy ion types and their LET values listed in Table 1 were used to develop this plot. It shows that the operational range of LET of this unit, as determined by the calibration process for the selected amplifier gain settings, is from 1.29 to $51.4 \mathrm{MeV} \cdot \mathrm{cm}^{2} / \mathrm{mg}$ for channels 8 to 127 , respectively (see Table 2). Channel 127 integrates all particle LETs greater than 51.418 
$\mathrm{MeV} \cdot \mathrm{cm}^{2} / \mathrm{mg}$. These parametric ranges can be increased or decreased to some degree by adjusting the amplifier gain.

Figure 2 also shows that the LET curve versus the HiLRS channel number is not a linear function for high LET values. This is clearly indicated in Table 2, which lists select channels and their corresponding LETs within channel ranges allocated to noise, proton induced events, and heavy ion events. However, the use of low LET alpha particles for calibration, combined with appropriate electronic adjustments, could be linear at those low LET values.

Regarding proton induced events, these are caused by secondary spallation, fractionation, or recoil products produced by proton interactions with atoms in or surrounding the sensitive volume of the detector nodes. Tests have indicated that it takes about $2 \mathrm{~K}$ to $5 \mathrm{~K}$ protons to generate a pulse event.

\section{DISCUSSION}

The results of the calibration shown in Table 2 indicate that the HiLRS response is not linear over this range of LET values. The curve fit to the data is an exponential function, as plotted in Figure 2. There are two possible locations in the system for this behavior to occur. One is the detector and the other the support electronics. The latter possibility can be eliminated since a test of the electronic response was performed and it was found to be linear. The most probable explanation for the non-linear behavior is the charge collection mechanism in the detector. Since there is no applied bias, the collection field is only due to the approximately 
$0.5 \mathrm{~V}$ drop across the junction. Therefore, it is possible that charge collection will not be linear in the HiLRS detector as the amplitude of charge generated increases with irradiation by particles of increasing LET. The non-linear collection will induce a non-linear distribution of voltage pulses across the bank of channels.

The flight-qualified HiLRS instrument delivered to the Air Force for STRV was specifically calibrated for use also as a Solar Proton Event (SEP) detector. Channels 8-20 were assigned for that purpose. This was considered important for two reasons: the type of trajectory, i.e. Geostationary Transfer Orbit (GTO), and time of launch in early 1999. The latter places the mission into the active phase of the solar cycle (solar max) when frequent SEPs occur, some extremely large. As for the trajectory, the spacecraft spends long intervals of time, around apogee, in regions of the earth's magnetosphere that are easily accessible to solar event protons. The HiLRS is expected to provide real-time information about the onset and the end of SEPs to the project and the other experimenters. The exposure of the instrument to the large proton fluxes encountered during passes through the South Atlantic Anomaly (SAA) at perigee is being ignored for that purpose since it does not interfere with the detection of SEPs in the outer regions of the magnetosphere.

\section{CONCLUSIONS}

A high-LET heavy ion radiation monitor has been fabricated, based on microelectronics principles. The design of the prototype HiLRS has successfully addressed the primary requirements of: small size $\left(4.5^{\prime \prime} \times 4.5^{\prime \prime} \times 0.5^{\prime \prime}\right)$, light weight (214 grams), low power $(0.5$ 
watt), rad-hard, low cost. The instrument measures energy deposited by solar and galactic cosmic rays and Van Allen belt trapped-proton nuclear interaction products. The detector, as calibrated, is well suited to map the high-LET radiation environment of satellites and aircraft.

\section{REFERENCES}

[1] McNulty, P.J., D.R. Roth, W.J. Beauvois, W.G. Abdel-Kader, and E.G. Stassinopoulos, "Microdosimetry in Space Using Microelectronic Circuits", NATO-ASI on Biological Effects and Physics of Solar and Galactic Cosmic Radiation, October 1991, Armacaco de Pera, Portugal, E. Swenberg, G. Hormeck, and E.G. Stassinopoulos, Editors. [2] Roth, D.R., P.J. McNulty, R.A. Reed, A. V. Thompson, and E.G. Stassinopoulos, "Solid State Microdosimeter for Spacecraft Applications", Second European Conference on Radiation and its Effects on Components and Systems (RADECS), September 1993, Saint-Malo, France, IEEE Catalog Number 93THD616-3, Jean-Luc Leray and Olivier Musseau, Editors. 


\begin{tabular}{|c|c|c|c|c|}
\hline \multicolumn{5}{|c|}{$\begin{array}{l}\text { Ions Incident on Sensitive Volume After } \\
\text { Passing Through } 5 \mu \mathrm{m} \text { EPI Layer }\end{array}$} \\
\hline$\underline{I O N}$ & $\frac{\text { ENERGY }}{\text { MeY) }}$ & $\frac{\mathrm{LET}}{\left.\mathrm{MeV} \cdot \mathrm{cm}^{2} / \mathrm{mg}\right)}$ & $\frac{\text { RANGE }}{(\mu \mathrm{m})}$ & $\frac{\text { ANGLE }}{\text { (deq) }}$ \\
\hline${ }^{6} \mathrm{C}-12$ & 98.3 & 1.45 & 182.5 & 0 \\
\hline${ }^{8} 0-16$ & 124.0 & 2.62 & 133.6 & 0 \\
\hline${ }^{14} \mathrm{Si}-28$ & 175.7 & 8.12 & 70.8 & 0 \\
\hline${ }^{17} \mathrm{Cl}-35$ & 196.4 & 11.8 & 58.5 & 0 \\
\hline${ }^{35} \mathrm{~B} 2-79$ & 245.9 & 37.9 & 32.2 & 0 \\
\hline
\end{tabular}

Table 1: Brookhaven Calibration 


\begin{tabular}{|c|c|c|}
\hline $\begin{array}{c}\text { Channel \# } \\
\text { N }\end{array}$ & Channel Description & $\begin{array}{c}\text { Particle LET } \\
\left(\mathrm{MeV} \cdot \mathrm{cm}^{\left.\frac{1}{2} \mathrm{mg}\right)}\right.\end{array}$ \\
\hline $0-7$ & Noise Channels & N/A \\
\hline 8 & First Proton Channel & 1.285 \\
20 & Last Proton Channel & 1.865 \\
\hline 21 & First Heavy Ion Channel & 1.923 \\
40 & Heavy Ion Channel & 3.466 \\
60 & Heavy Ion Channel & 6.443 \\
80 & Heavy Ion Channel & 11.977 \\
100 & Heavy Ion Channel & 22.265 \\
115 & Heavy Ion Channel & 35.445 \\
120 & Heavy Ion Channel & 41.388 \\
125 & Heavy Ion Channel & 48.327 \\
126 & Last Discrete Heavy Ion Channel & 48.849 \\
\hline 127 & Integrated Heavy Ion Channel & $\geq 51.418$ \\
\hline
\end{tabular}

Table 2: Brookhaven Heavy Ion Calibration Results

$$
\left(\mathrm{LET}=1.003 * \mathrm{e}^{0.031 \times \mathrm{N}}\right)
$$


The High-LET Radiation Spectrometer Block Diagram

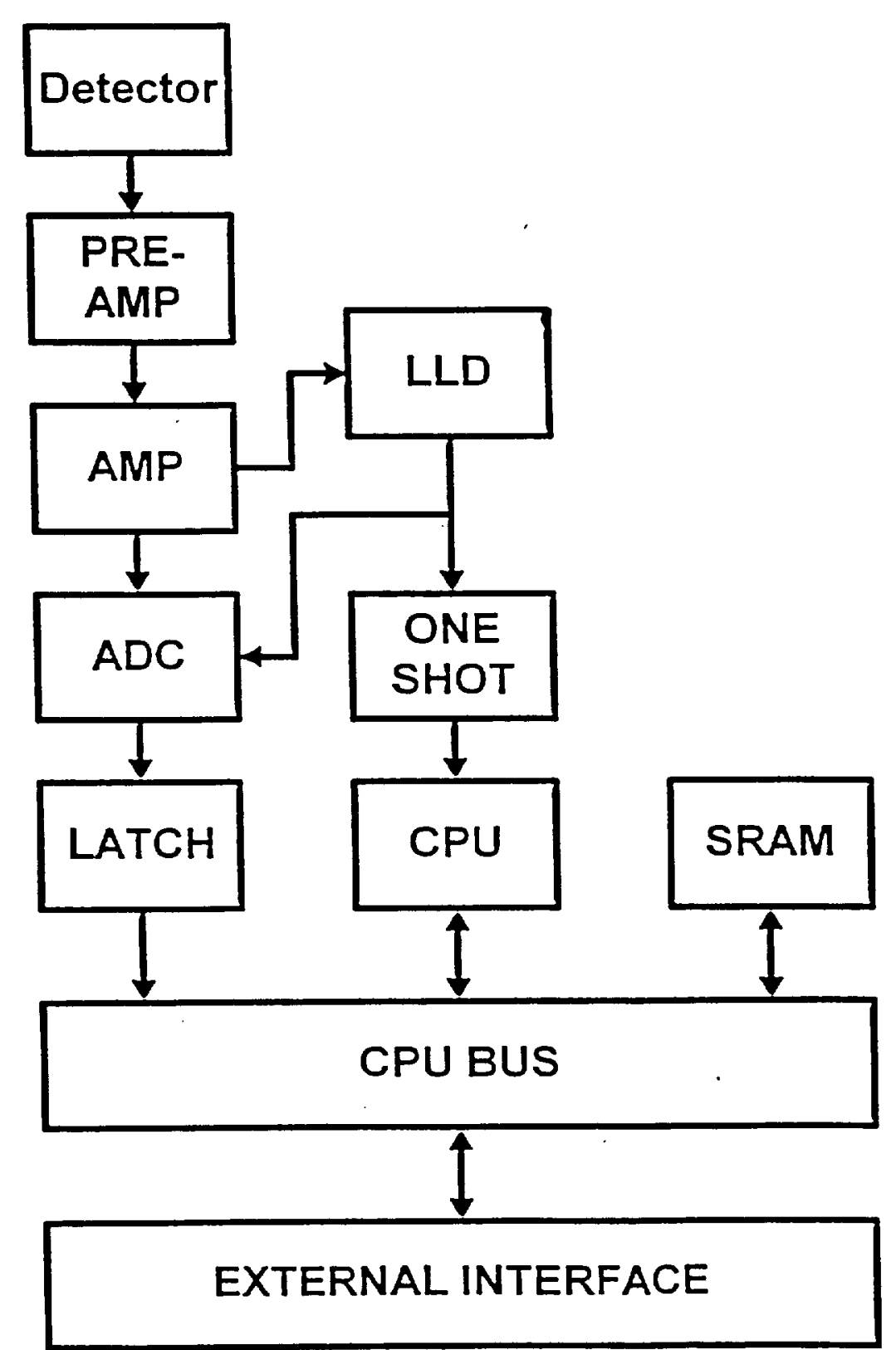

Figure 1 


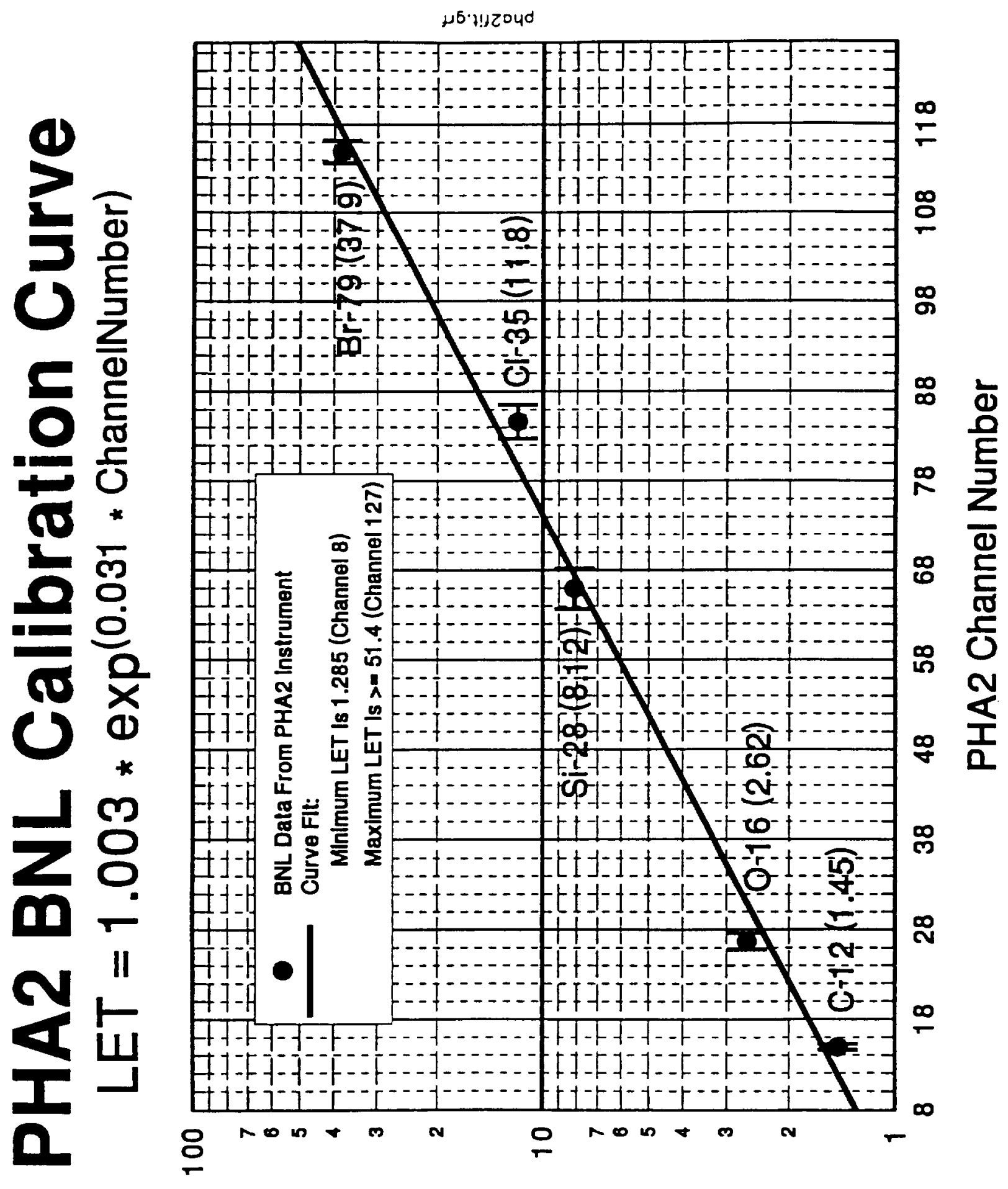

$(6 ய / 乙 ய 0 * \wedge \partial W) \perp \exists า$ 